\title{
Grupo focal como técnica de coleta de dados na pesquisa em enfermagem: Um relato de experiência
}

\author{
Focal group as a data collection technique in nursing research: An experience report
}

Focal group como técnica de recopilación de daos em la investigación em enfermería: Un informe de experiencia

Alice de Andrade Santos ${ }^{1,2}$, Larissa Chaves Pedreira ${ }^{1}$, Raniele Araújo de Freitas ${ }^{1}$, Nildete Pereira Gomes $^{1}$, Tânia Maria de Oliva Menezes ${ }^{1}$, Luna Vitoria Cajé Moura ${ }^{1,3}$.

\section{RESUMO}

Objetivo: Relatar a experiência do Grupo Focal como técnica de coleta de dados na pesquisa em enfermagem. Relato da experiência: Foram realizados três encontros em salas reservadas, com duração de 90 minutos, entre maio e junho de 2016. Ao utilizar a técnica de grupo focal, os desafios foram: dificuldade em reunir os colaboradores, necessidade de conhecimento prévio da técnica por parte da moderadora, o que demandou maior tempo de preparo porém garantiu uma mediação mais coerente, e o desafio da limitação do número de participantes, que era por vezes o mínimo indicado para a utilização da referente técnica. Este número teve como causa fatores relacionados à natureza dos vínculos empregatícios e das atividades extenuantes realizadas pelos participantes. Percebeu-se a necessidade de maior exploração de saberes sobre esta técnica de coleta de dados, bem como estímulo dos profissionais em participar de metodologias ativas. Durante os encontros houve envolvimento proativo dos participantes e percepção por eles da importância destes momentos. Considerações finais: A utilização do Grupo Focal gerou aprendizados e experiências para manejo desta técnica, e apesar das dificuldades, mostrou-se como uma experiência exitosa. Entretanto, ressalta-se a escassez de estudos sobre o uso do Grupo Focal nas pesquisas em enfermagem.

Palavras-chave: Grupos focais, Pesquisa em enfermagem, Aprendizagem baseada na experiência.

\begin{abstract}
Objective: To report the experience of the Focus Group as a data collection technique in nursing research. Experience report: Three meetings were held in 90-minute private rooms between May and June 2016. Using the focus group technique, the challenges were: difficulty in bringing employees together, prior knowledge of the technique by the moderator, which required more preparation time but ensured a more coherent mediation, and the challenge of limiting the number of participants, which was sometimes the minimum indicated for the use of this technique. This number was caused by factors related to the nature of employment relationships and strenuous activities performed by participants. There was a need for further exploration of knowledge about this data collection technique, as well as encouragement of professionals to participate in active methodologies. During the meetings there was proactive involvement of the participants and their perception of the importance of these moments. Final considerations: The use of the Focus Group generated learning and experiences for the management of this technique, and despite the difficulties, it proved to be a successful experience. However, there is a scarcity of studies on the use of the Focus Group in nursing research.
\end{abstract}

Keywords: Focus groups, Nursing research, Experience based learning.

\footnotetext{
1 Universidade Federal da Bahia. Salvador- Bahia. *E-mail: lice_andrade1@hotmail.com

2 Centro Universitário UniRuy Wyden, Salvador- Bahia.

${ }^{3}$ Faculdade Adventista da Bahia, Cachoeira-Bahia.
} 


\section{RESUMEN}

Objetivo: Informar la experiencia del Grupo de enfoque como técnica de recolección de datos en la investigación de enfermería. Informe de experiencia: se llevaron a cabo tres reuniones en salas privadas de 90 minutos entre mayo y junio de 2016. Utilizando la técnica del grupo focal, los desafíos fueron: dificultad para reunir a los empleados, conocimiento previo de la técnica por el moderador, que requirió más tiempo de preparación, pero aseguró una mediación más coherente, y el desafío de limitar el número de participantes, que a veces era el mínimo indicado para el uso de esta técnica. Este número fue causado por factores relacionados con la naturaleza de las relaciones laborales y las actividades extenuantes realizadas por los participantes. Era necesario seguir explorando el conocimiento sobre esta técnica de recopilación de datos, así como alentar a los profesionales a participar en metodologías activas. Durante las reuniones hubo una participación proactiva de los participantes y su percepción de la importancia de estos momentos. Consideraciones finales: El uso del Grupo de enfoque generó aprendizaje y experiencias para el manejo de esta técnica y, a pesar de las dificultades, resultó ser una experiencia exitosa. Sin embargo, existe una escasez de estudios sobre el uso del Grupo de enfoque en la investigación de enfermería.

Palabras clave: Grupos focales, Investigación en enfermería, Aprendizaje basado en la experiencia.

\section{INTRODUÇÃO}

O Grupo Focal (GF) é compreendido como uma técnica de investigação da metodologia qualitativa de caráter descritivo, que busca apreender opiniões e atitudes dos entrevistados em relação ao objeto pretendido da pesquisa, possibilitando a integração do grupo estudado; estimulando o pensamento crítico e reflexivo sobre o objeto, a partir do debate proporcionado; respostas coerentes e novas ideias. Esse tipo de técnica de investigação permite que o pesquisador conheça, in loco, as percepções dos participantes de forma detalhada (SOARES Ml, et al., 2016).

A técnica de GF, empregada nas pesquisas qualitativas, se constitui como um recurso cada vez mais utilizado para coleta de dados, principalmente quando se refere a situações nas quais o pesquisador pretende apurar uma temática ou evento sob uma ótica coletiva (MUNARETTO LF, et al., 2013).

Como técnica de coleta na pesquisa, o GF possibilita investigar a inserção de participantes em qualquer universo da área da saúde, permitindo que adentrem em um momento de contextualização, reflexão e análise do objeto investigado, gerando pontos de vista variados, opiniões, críticas e sugestões (TRAD LB, 2009).

No campo da saúde, a Enfermagem foi pioneira na utilização desta técnica de coleta de dados, e mantém sua contribuição em uma tendência ascendente, tanto quantitativa quanto qualitativamente, na produção de conhecimento (KINALSKI DF, et.al., 2017). A empatia e a abordagem participativa, que devem estar presentes no ato de cuidar ofertado pela equipe de enfermagem, são iniciativas que se fazem imprescindíveis também na aplicação da técnica de grupo focal como foi percebida a partir da experiência relatada neste estudo.

Ademais, esta técnica permite compreender o ser humano em sua complexidade e profundidade, proporciona a aproximação entre o ensino e a prática, e desenvolve uma assistência em saúde por meio de vivências, experiências e relações sociais (CUESTA BC, 2010; KERR LS, 2013 e MEDEIROS M.,2012). Por esta razão, se mostra como importante ferramenta para a investigação em enfermagem para práticas baseadas em evidências e que reflitam a realidade vivenciada pela categoria profissional.

Ainda se tratando da enfermagem, neste campo a técnica de GF tem sido explorada comumente pelos pesquisadores para a investigação de estudos de natureza qualitativa. Sendo assim, pode ser uma estratégia largamente utilizada pelos profissionais de saúde, compondo um mecanismo motivador de discussões ativas, oportunidade de análise detalhada de expressões, percepções e condição de entendimento das respostas ampliadas e reflexivas (GODOY MH e MUNARI DB, 2006).

Como uma técnica de coleta de ampla utilidade nas pesquisas qualitativas, sua execução exige rigor metodológico, planejamento rigoroso e preparo do pesquisador, que assume importante papel de moderador. 
Dessa forma, entende-se que relatos de experiência podem contribuir para que outros pesquisadores conheçam esta técnica, e utilize-a de modo direcionado para o alcance do objetivo da pesquisa. Assim, este artigo tem como objetivo relatar a experiência da utilização do GF como técnica de coleta de dados na pesquisa em enfermagem.

\section{RELATO DE EXPERIÊNCIA}

\section{Operacionalização do Grupo Focal}

A coleta de dados foi realizada entre maio e junho de 2016, na unidade de emergência do hospital de referência para doenças cerebrovasculares, tendo como participantes 16 enfermeiros classificadores, selecionados a partir dos seguintes critérios de inclusão: exercer atividades fixas no setor de acolhimento com classificação de risco (ACCR), ter no mínimo seis meses de experiência no setor. Como critérios de exclusão estar de licença médica no período da coleta de dados.

A realização do GF contou com o auxílio de duas estudantes de graduação, bolsistas de iniciação científica, duas mestrandas e quatro integrantes do Núcleo de Estudos e Pesquisas do Idoso (NESPI), todos da Escola de Enfermagem da Universidade Federal da Bahia (EEUFBA).

Inicialmente, foram realizadas cinco reuniões na EEUFBA, com o objetivo de organizar as sessões do GF e a participação de cada membro da equipe de apoio, que foram divididos nas funções de: receber os participantes, entregar os crachás de identificação por número e o termo de esclarecimento e consentimento para assinatura, gravar as falas, controlar o tempo, observar e anotar as reações, servir água e café e organizar um lanche para o final. Essa organização foi necessária, para que a pesquisadora pudesse se concentrar na condução as discussões com mais liberdade e sem interrupções, aproveitando todas as falas.

Perfil dos participantes da pesquisa

Os participantes da pesquisa tinham idade entre 24 e 51 anos, sendo 14 mulheres e dois homens, a maioria autodeclarados negros. Grande parte dos participantes relatou experiência profissional na unidade de emergência há mais de um ano. Referiram ainda exercerem carga horária de trabalho acima de 36 horas semanais, e salário variando entre dois mil e cinco mil reais. Dos 16 entrevistados, sete cursaram especialização, sendo três na área da emergência e os demais em gestão hospitalar, enfermagem cardiológica e hemodinâmica, enfermagem do trabalho e saúde pública.

Para motivar a participação do grupo, entendendo também que estes estavam passando por um momento difícil na instituição, com atrasos nos salários, insatisfação em realizar atividades laborais na unidade, devido à alta demanda de atendimento e as precariedades do serviço público, foi preciso utilizar de algumas estratégias como: viabilizar um momento após o encontro do GF com coffee break, para que os integrantes pudessem socializar, relaxar e entender a importância da participação de todos na pesquisa. Além disso, foi combinado com a coordenação a liberação em escala de seis horas na carga horária mensal, visto que as reuniões aconteciam no próprio hospital, fora do horário de trabalho, e muitos se deslocavam de suas residências. Tais estratégias foram necessárias, pois não havia possibilidade de o GF reunir os enfermeiros em seu horário de trabalho, visto a dinâmica de trabalho do setor.

\section{Quantidade e duração do grupo focal}

Foram realizados três encontros de grupo de discussão sobre a temática ACCR à pessoa idosa com suspeita de acidente vascular cerebral (AVC), optando-se por abordar pessoas distintas utilizando as mesmas questões norteadoras em cada encontro. A escolha por três dias correspondia a divisão exata do número de participantes.

A duração de cada encontro foi de 90 minutos, com a intenção de evitar discursos recorrentes e desnecessários, sendo marcada data e horários previstos conforme disponibilidade em escala dos participantes, e acordo estabelecido previamente com a coordenação de enfermagem da unidade de emergência. 


\section{Considerações gerais sobre a aplicação da técnica}

Ao iniciar as discussões, a moderadora aplicava os seguintes questionamentos utilizando os pronomes interrogativos: "como", "o que" e "quais". "Como ocorre o ACCR a pessoa idosa com suspeita AVC nesta unidade de referência?"; "O que você entende que deve acontecer nesse tipo de acolhimento?" e "Quais dificuldades e facilidades você vivência no acolhimento a este público"?

Conseguinte, a exposição das perguntas poderosas, os profissionais eram estimulados a discorrer sobre suas concepções acerca das práticas de acolhimento executadas nessa instituição. Esse momento foi marcado por euforia, estresses e desabafos dos problemas enfrentados no cotidiano da unidade, sendo necessária a participação mais enérgica da moderadora para o direcionamento dos discursos, no intuito de organizar as falas e obter relatos detalhados de caráter individual e coletivo.

Ao término de cada encontro, realizava-se uma avaliação da opinião dos colaboradores sobre o uso do GF para coleta de dados. Todavia, incumbe elucidar que, a moderadora e as observadoras reuniam-se ao término de cada sessão, com a finalidade de realizar uma avaliação sobre o momento da coleta, esse comportamento permitia a troca de informações sobre a atuação dos pesquisadores e depoentes.

De um modo geral, a técnica elucidou as fragilidades da unidade pesquisada de acordo com os relatos, e foi caracterizada como uma oportunidade de expressão, uma vez que, na opinião dos enfermeiros, permitia a transparência de suas opiniões e ao mesmo tempo, a analise imparcial da moderadora frente ao envolvimento dos participantes, proporcionando momentos de exposição e trocas de experiências entre todos envolvidos.

\section{DISCUSSÃO}

O momento do primeiro encontro de GF foi marcado pela expectativa da pesquisadora e do seu grupo de apoio para receber seis participantes, conforme combinação anterior. Entretanto, compareceram no primeiro dia apenas quatro participantes, no segundo dia cinco e, no terceiro, sete. Embora o número de participantes não fosse o que estava previamente planejado, foi dado início às atividades, tomando-se com parâmetro pesquisa que corrobora, a quantidade mínima de quatro participantes por sessão (DALL'AGNOL CM, et al., 2012).

Conseguinte, a principal característica de um GF é ser organizado com um pequeno número de pessoas, podendo variar entre cinco a 12 participantes (VON SEGGERN M e YOUNG NJ, 2003). Outros estudos pontuam que, em termo de composição de GF, a literatura é mista em relação às opiniões sobre tamanho ideal, e diferentes pesquisadores refutam a afrimativa supracitada e observaram intervalos de tamanho de quatro e seis a oito participantes (BODDY C, 2005; MASADEH MA 2012; MARCZAK M e SEWELL M, 2007).

A respeito da participação do moderador durante a aplicação da técnica de GF, entende-se que o GF permite ao moderador (a) participar da pesquisa, observando atentamente falas e expressões, bem como redirecionar as discussões no momento que as falas evadem da temática proposta (MASADEH MA, 2012).

Sobre a avaliação que deve ser realizada frequentemente durante a aplicação da técnica, estudo corrobora, que esta deve ser realizada para elucidar algumas percepções referida pelo grupo, bem como momentos de autorreflexões críticas acerca dos dados coletados e desempenho dos pesquisadores, originando propriedade para as abordagens dos encontros subsequentes (ASCHIDAMINI IM e SAUPE R, 2014).

O estudo evidenciou a importância na busca do conhecimento prévio frente às possíveis potencialidades e limitações apresentadas pelo GF, durante o planejamento e no decorrer da execução da fase de coleta de dados. Entende-se, como fator dificultoso, a necessidade de realizar os encontros, com exigência da quantidade mínima do número participantes, conforme recomendação da literatura nacional, no que concerne ao número de participantes no GF, foi evidenciado na literatura uma oscilação entre seis a 15 (TRAD LB, 2009). Diante desse dado, foi necessário a busca de dados internacionais, para o embasamento científico 
acerca da quantidade mínima de participantes. Sendo assim, sugere-se ampliações das discussões e novos estudos sobre o manejo da técnica do GF, nas pesquisas da área de saúde do Brasil.

Conseguinte, acerca dos sujeitos abordados, observou-se que o debate permitiu elucidar déficit no sistema de referência e contra referência; dificuldades no processo de trabalho e fragilidades no que concerne as competências e habilidades para o manuseio do ACCR. Estudo corrobora, que a utilização do GF no processo de investigação de dados, proporciona aproximação, relatos de experiência e discussões entre os profissionais investigados, esse momento ímpar aponta algumas sugestões para o enfrentamento das complexidades diárias (VIEIRA CM, et al., 2013).

A versatilidade da técnica de GF atrelada a utilização das dinâmicas de grupo, pode permitir momentos de desabafos como: choros, insegurança e medos por partes dos sujeitos investigados, e aos pesquisadores a percepção sérias fragilidades emocionais durante relatos da prática de classificação de risco. Pesquisa elucida, que aplicação da técnica de GF proporciona um espaço fecundo para exposição das opiniões, sentimentos e relatos durante o desenvolvimento dos grupos de discussão (NETO OC, et.al., 2012).

No entanto, algumas questões de cunho pessoal dos participantes, como insegurança ao executar o ACCR, insatisfação remuneração mensal e/ou vínculo empregatício terceirizado e discordâncias da gestão local, interferiram nas discussões, sendo necessária uma condução dinâmica e atrativa da moderadora a fim de executar a técnica em ambiente agradável e obter informações genuínas. Neste aspeto, pesquisa desvela que o(a) moderador (a) deve apresentar a competência de executar a dinâmica de condução do grupo com segurança e firmeza, transmitindo a transparência direcionada ao objeto do estudo e intervindo em conflitos desfocados, computando com a colaboração do observador (a) no registro de elementos subjetivos dos participantes (GATTI BA, 2005).

Entretanto, a utilização do GF oportunizou a pesquisadora e a equipe de apoio ao desenvolvimento de habilidades, a saber: organização logística, prática no controle das discussões a respeito do comprimento das regras de convívio e moderação das discussões, para manter o foco de êxito durante a coleta de dados. Ademais, o objetivo proposto pela pesquisa foi alcançado, e as discussões permitiram uma reflexão crítica do universo investigado por parte da moderadora, participantes e do grupo de apoio, presente em todos os encontros.

Ademais, a pesquisa apresentou algumas limitações durante a operacionalização do GF, no entanto, obteve-se um resultado satisfatório e aceitação entre os participantes e pesquisadores, demarcando uma possibilidade concreta de optar por essa técnica de coleta de dados, em pesquisa qualitativa no campo da enfermagem.

\section{REFERÊNCIAS}

1. ASCHIDAMINI IM, SAUPE R. Grupo focal - estratégia metodológica qualitativa: um ensaio teórico. Cogitare Enfermagem. 2014; 9:9-14.

2. CUESTA BC. La investigación cualitativa y El desarrollo Del conocimiento en enfermería. Texto Contexto Enferm, Florianópolis, 2010 Out-Dez; 19(4): 762-6.

3. DALL'AGNOL CM, et al. A noção de tarefa nos grupos focais. Revista Gaúcha Enfermagem. Porto Alegre (RS). 2012;33(1):186-90.

4. GATTI BA. Grupo focal na pesquisa em ciências sociais e humanas. Brasília: Liber Livro; 2005. 75p.

5. GODOY MH, MUNARI DB. Review of scientific literature on the use of group activities in nursing work in Brazil: 1980 to 2003. Revista Latino-Americana de Enfermagem. 2006; 14(5):786-802.

6. KERR LS, KC. A pesquisa qualitativa em saúde Rev Rene. 2013; 14(6):1061-3

7. KINALSKI DF, et al. Focus group on qualitative research: experience report. Rev Bras Enferm. 2017;70(2):424-9.

8. MARCZAK M, SEWELL M. Using focus groups for evaluation. Cybernet Evaluation. Tuscon, AZ: The University of Arizona 2007.

9. MASADEH MA. Focus Group: Reviews and Practices. International Journal of Applied Science and Technology. 2012;2(10).

10. MEDEIROS M. Thinking about qualitative research. Rev Eletr Enf. 2012;14(2):224-5. 
11. MUNARETTO LF, et al. A study on the characteristics of the Delphi method and focus group as techniques to obtain data in exploratory research. Revista de Administração da UFSM.2013;(6):1:9-24.

12. NETO OC, et al. Grupos focais e Pesquisa Social Qualitativa: o debate orientando como técnica de investigação. In: XIII Encontro da Associação Brasileira de Estudos Populacionais. Ouro Preto; 2012. p. 1-26.

13. SOARES MI, et al. Technique of focus group in qualitative data collection: experience report. Revista Mineira de Enfermagem. 2016; 20 e 942.

14. TRAD LB. Grupos focais: conceitos, procedimentos e reflexões baseadas em experiências com o uso da técnica em pesquisas de saúde. Physis: Revista de saúde coletiva, 2009; (19):777-96.

15. VON SEGGERN M, YOUNG NJ. The focus group method in libraries: issues relating to process and data analysis. Services Review. 2003; 31(3):272-84.

16. VIEIRA CM, et al. Aplicação da técnica de grupo focal em pesquisa da Rede-SANS sobre as ações de alimentação e nutrição na atenção básica em saúde. Cadernos Saúde Coletiva, 2013. 